UMN-TH-1213-93

TPI-MINN-93/48-T

hep-ph/9401208

December 1993

\title{
Protecting the Primordial Baryon Asymmetry From Erasure by Sphalerons
}

\author{
James M. Cline \\ Kimmo Kainulainen \\ and \\ Keith A. Olive \\ School of Physics and Astronomy, University of Minnesota \\ Minneapolis, MN 55455, USA
}

\begin{abstract}
If the baryon asymmetry of the universe was created at the GUT scale, sphalerons together with exotic sources of $(B-L)$-violation could have erased it, unless the latter satisfy stringent bounds. We elaborate on how the small Yukawa coupling of the electron drastically weakens previous estimates of these bounds.
\end{abstract}




\section{Introduction}

The first proposals to explain the baryon asymmetry of the universe suggested that baryogenesis occurred at the very high temperatures characteristic of the scale of grand unification. While there has been great interest in generating baryons at the electroweak scale, the feasibility of this approach remains uncertain, whereas baryon-production in grand unified theories can be calculated fairly reliably in the framework of a given GUT. However there are many potential opportunities for a baryon asymmetry produced at $10^{16} \mathrm{GeV}$ to be destroyed later on, which leads to interesting constraints on the coefficients of operators that violate baryon or lepton number.

A baryon asymmetry generated at the GUT scale will survive only if baryon number violating interactions are subsequently out of thermal equilibrium. In most $S U(5)$ grand unification schemes, any baryon asymmetry generated will be accompanied by a lepton asymmetry such that $B-L=0$. Non-perturbative electroweak interactions via sphalerons also violate baryon and lepton (actually $B+L$ ) number at high temperatures [1] while perserving $B-L$. As such, the GUT-scale produced baryon asymmetry will be erased [2] if sphaleron interactions are in thermal equilibrium, as they are expected to be at temperatures between $\sim 10^{2}$ and $10^{12} \mathrm{GeV}$. By the same token, any GUT-scale baryon asymmetry with a net $B-L$ asymmetry will be preserved.

If in addition to the sphaleron interactions there was another baryon or lepton number violating interaction in a linear combination other than $B+L$ and in thermal equilibrium, all net baryon and lepton numbers would be erased. Such was the case encountered by Fukugita and Yanagida (FY) [3] when considering a simple extension to the standard electroweak model to allow for Majorana neutrino masses. In order to maintain a net baryon asymmetry, this source of lepton number violation is constrained to be out of equilibrium at the times when sphalerons are in thermal equilibrium. This constraint was then translated into a bound on the neutrino mass.

FY derived the conservative bound $m_{\nu} \gtrsim 50 \mathrm{keV}$, assuming only that sphalerons are in

equilibrium at $T \sim 10^{2} \mathrm{GeV}$. It was subsequently argued that this constratint could be made substantially stronger because sphalerons are in equilibrium at significantly higher 
temperatures as well [4]. These arguments were also extended to other sources of baryon and lepton number violation [5, 6].

In a previous paper [7] we pointed out that the constraints are dramatically weakened when one realizes that right-handed electrons can protect the baryon asymmetry in cases where it would otherwise have been destroyed, so long as the interactions of the operators in question fall out of equilibrium before the interactions of $e_{R}$ with $e_{L}$ and Higgs bosons come into equilibrium. The idea that the weakness of $e_{R^{-}} e_{L}$-Higgs interactions might have something to do with preserving the baryon asymmetry from sphalerons and other $B$ - or $L$-violating effects was originally considered by the authors of ref. [8] (CDEO), for the initial condition that $B-L=0$. Their goal was to determine whether $e_{R} \leftrightarrow e_{L}$ equilibrium might be established so late that sphaleron interactions would not have enough time to nullify the baryon asymmetry.

The argument for the importance of $e_{R}$ decoupling is intuitively very simple and was presented in a rudimentary form in our previous work [7]. Since we feel that the idea has far-reaching consequences, it seems worthwhile to rederive the earlier results in a more accurate and rigorous fashion. Moreover there is a variety of special cases for the initial conditions or the depletion at intermediate scales of the $B$ and $L$ asymmetries, each with its own subtleties, the discussion of which was beyond the scope of the first paper. Filling these gaps is the goal of the present work.

In what follows we will develop the full network of Boltzmann equations necessary to solve for the baryon asymmetry through the thermalization of $e_{R}$. In section 3, we derive the rates for decays and inverse decays as well as scatterings to determine the $e_{R}$ decoupling temperature. We then consider the case with $B-L=0$ as did CDEO, in section 4. Although their result indicated that the baryon asymmetry was not preserved in this case, the final baryon asymmetry is exponentially sensitive to changes in reaction rates, and we have undertaken a careful reanalysis of some effects which were treated too roughly by CDEO. In particular, we have included the effects of scatterings. Qualitatively, our results confirm those earier conclusions. In section 5 , we generalize to the case where $B-L \neq 0$ and describe several mechanisms in which a baryon asymmetry can be preserved from sphaleron effects. Our conclusions will be given in section 6 . 


\section{The network of Boltzmann equations}

In this paper we explore the consequences of the fact that the right-handed chiral electrons $e_{R}$ remain out of equilibrium to suprisingly low temperatures in the early universe. This phenomenon arises simply due to the smallness of the electron mass or its Yukawa coupling, $h_{e} \simeq 2.94 \times 10^{-6}$. At temperatures above the electroweak phase transition electrons are massless, or more accurately, different chiral states have only chirality-conserving temperature-dependent masses, so that the only processes that change lepton chirality are interactions with Higgs bosons. The lepton chirality-changing rate is therefore proportional to the small number $h_{e}^{2}$. The smallness of the $e_{R} e_{L}$ equilibration temperature $T_{*}$ is important because the equilibrium conditions in the primieval plasma are different above and below $T_{*}$. This has far-reaching consequences for the regeneration of the baryon asymmetry and leads to a considerable relaxation of bounds imposed on various exotic interactions to preserve the primordial baryon asymmetry.

It was noted in CDEO that in the particular case of a universe with initial $B-L=0$ the final baryon asymmetry is exponentially sensitive to the value of the rate of the chirality changing interactions. In such a case special care must be taken in computing the thermally averaged rates and also in deriving the evolution equations for the asymmetries from the Boltzmann equations for the particle densities. Indeed the evolution equations found in the literature are often somewhat inaccurate in their treatment of the relevant collision terms in the Boltzmann equations.

In this section we will carefully derive evolution equations for the particle asymmetries in the early universe during the time when $e_{R} \leftrightarrow e_{L}$ transitions come into equilibrium. In general these equations couple all particle species present in the heat bath and involve a very large number of reaction rates. Fortunately a natural hierarchy exists between different reactions; various interaction processes can be divided into 'fast' or 'slow' depending on how their rates compare to the Hubble expansion rate of the universe $H$ at the timescale

\footnotetext{
${ }^{1}$ For the quarks, QCD instanton interactions also violate chirality, but it is easy to show that these give no new conditions on the chemical potentials; they are consistent with the constraints associated with Higgs boson interactions.
} 


\begin{tabular}{|l|c|}
\hline \hline (Inverse) Decays & $H^{*} \leftrightarrow e_{R} L_{e}{ }^{c}$ \\
\hline Gauge scattering & $e_{R} H \leftrightarrow L_{e} A$ \\
\cline { 2 - 2 } & $e_{R} A \leftrightarrow L_{e} H^{*}$ \\
\cline { 2 - 2 } & $e_{R} L_{e}{ }^{c} \leftrightarrow A H^{*}$ \\
\hline Fermion scattering & $e_{R} L_{f} \leftrightarrow L_{e} f_{R}$ \\
\cline { 2 - 2 } & $e_{R} L_{f}^{c} \leftrightarrow L_{e}{ }^{c} f_{R}$ \\
\cline { 2 - 2 } & $e_{R} L_{e}{ }^{c} \leftrightarrow L_{f}^{c} f_{R}$ \\
\hline \hline \multirow{2}{*}{$\begin{array}{l}\text { Electron lepton } \\
\text { number violation }\end{array}$} & $e_{L} L_{\ell} \leftrightarrow h^{-} H$ \\
\hline \hline
\end{tabular}

Table 1: Shown are all tree level interactions that change the chirality of electrons and also a particular type of electron lepton number violation. $H$ is the Higgs doublet, with components $\left(h^{+}, h\right)^{T} . L_{i}$ refers to either of the members in a left-handed doublet; $f$ represents all fermions but $\ell$ is restricted to the leptons. $A$ can be either the $B$ or the $W$ gauge field.

of interest. A process is called fast only if its rate is much bigger than $H$, otherwise it is considered to be slow. Fast processes need not be treated in detail, but instead, their effect can be accounted for by an appropriate set of equilibrium conditions between chemical potentials and certain conservation laws that restrict the explicit equations governing the slow processes.

Examples of fast processes are the various electroweak interactions connecting fermions in the weak isospin doublets, $W^{-} \leftrightarrow \overline{\nu_{e}} e^{-}, \bar{u} d$, etc., and the interactions mediated by the electroweak anomaly, i.e. the sphaleron interactions [1]. Naturally, all interactions that change the chirality of electrons are taken to be slow, but there can also be others, due to new physics. In Table 1 we have collected all slow processes that are relevant to our considerations in this section. For illustrative purposies we also show an electron lepton number violating interaction, which we will study in detail in section 5 below.

Evolution equations for the asymmetries in the $e_{R}$ and $e_{L}$ species can be written down in a quite general manner, but to make the equations close typically requires some additional constraint that is specific to a given system. The problem shows up, for example, in the appearance of the Higgs chemical potential $\mu_{0}$ in the evolution equations; the expression 
for $\mu_{0}$ in terms of $\mu_{e_{R}}$ and $\mu_{e_{L}}$, if it can be given, depends on the particular model. It is also clear that if any particle number other than $e_{L}$ or $e_{R}$ numbers was softly violated by some new exotic slow interaction, then additional equations governing the evolution of the corresponding asymmetry would be needed. Here we will restrict ourselves to the case where at most $e_{L}$ violation (in addition to $e_{R^{-}} e_{L}$ equilibration) is tracked in detail, which includes the special situations that we study in sections 4 and 5 . Our derivation however will address all the subtleties needed to derive evolution equations for any other system of interest.

\subsection{Equilibrium conditions}

Let us first consider some of the equilibrium conditions established by the fast processes. Because sphaleron interactions fall out of equilibrium very quickly below the electoweak phase transition temperature [9] and because the sphaleron reprocessing of the changing $e_{R}$ asymmetry is crucial for our considerations in this paper, it is sufficient for us to consider the evolution equations only at temperatures above the phase transition. In the high temperature phase, the electroweak interactions mediated by the charged gauge bosons and scalars force the chemical potentials of quarks and leptons to obey the equilibrium conditions [ [4]

$$
\begin{aligned}
\mu_{d_{L}} & =\mu_{u_{L}} & \mu_{e_{L}} & =\mu_{\nu_{e}} \\
\mu_{u_{R}}-\mu_{u_{L}} & =\mu_{0} & \mu_{\ell_{L}} & =\mu_{\nu_{\ell}} \\
\mu_{d_{R}}-\mu_{d_{L}} & =-\mu_{0} & \mu_{\ell_{R}}-\mu_{\ell_{L}} & =-\mu_{0} .
\end{aligned}
$$

Here the labels $u$ and $d$ refer to all quarks with weak isospin $+1 / 2$ and $-1 / 2$ respectively, and $\ell$ refers to the muon and tau lepton; the chirality equilibrium equation is not assumed to hold for electrons. $\mu_{0}$ is the chemical potential of the Higgs field. We have assumed that the universe was initially isospin-neutral, f implying that the $W$ chemical potential,

\footnotetext{
${ }^{2}$ In contrast to the charge neutrality of the Universe, which is proved to a very high degree by observations, there is no requirement for the universe initially to have had zero net isospin $Q_{3}$; any primordial isospin would have vanished immediately after the phase transition leaving no observable trace today. However, it is improbable that such an asymmetry could have been dynamically generated because $Q_{3}$ corresponds to a gauge symmetry. Moreover, any initial value for it would have been washed away by inflation. We therefore adopt the value $Q_{3}=0$ as the most natural choice, albeit not an absolutely necessary.
} 
which would otherwise appear in eq. (11) above, is zero at high temperatures [4]. Note also that due to the flavour mixing in the quark sector all quark flavours have the same chemical potential. Equilibrium sphaleron interactions impose a further condition between the chemical potentials of quarks and leptons

$$
9 \mu_{u_{L}}+\mu_{e_{L}}+\sum_{\ell=\mu, \tau} \mu_{\ell_{L}}=0
$$

and the charge neutrality of the universe leads to

$$
Q=6 \mu_{u_{L}}+13 \mu_{0}-\mu_{e_{L}}-\mu_{e_{R}}-2 \sum_{\ell=\mu, \tau} \mu_{\ell_{L}}=0
$$

Equations (2-3) contain six unknown parameters. In addition to (2-3) we will always have four additional conditions on the chemical potentials so that the system has a unique solution. Assuming that there were no new interactions, then well above $T_{*}$ we would have the following conservation laws:[?

$$
\begin{aligned}
\frac{1}{3} B-L_{e} & =C_{e, p} \\
\frac{1}{3} B-L_{\mu} & =C_{\mu, p} \\
\frac{1}{3} B-L_{\tau} & =C_{\tau, p} \\
L_{e_{R}} & =C_{e_{R}, p}
\end{aligned}
$$

where $L_{f} \equiv L_{f_{L}}+L_{f_{R}}+L_{\nu_{f}}$ and the subscripts $p$ in the constants $C$ indicate that they are the primordial values. The connection between the asymmetries and the chemical potentials is

$$
L_{f} \equiv \frac{n_{f}-n_{f^{c}}}{s} \simeq \frac{15 c}{4 \pi^{2} g_{*}} \frac{\mu_{f}}{T}+O\left(\left(\mu_{f} / T\right)^{3}\right),
$$

where $c$ is 1 for fermions and 2 for bosons and $s=\frac{2 \pi^{2}}{45} g_{*}(T) T^{3}$ is the entropy density of the universe.

\footnotetext{
${ }^{3}$ We warn the reader that the symbol $L_{e}$ can mean the electron lepton doublet field, the asymmetry in electron lepton number, or the $\mathrm{U}(1)$ symmetry for electron lepton number, depending on the context. A similar warning applies for $L_{\mu}$ and $L_{\tau}$.
} 
If any of the conservation laws (4) are violated by exotic fast interactions, then they must be replaced by new equilibrium conditions. Indeed, the situation well below $T_{*}$ provides an example of how this works. There the $e_{L}-e_{R}$ transitions violate $e_{R}$ number strongly, so that the last equation in (푸) is replaced by the equilibrium condition $\mu_{e_{R}}-\mu_{e_{L}}=-\mu_{0}$. On the other hand, if some of the conservation laws are softly violated, so that around $T_{*}$ the rates of relevant interactions are comparable to the expansion rate, then explicit equations must be written for the asymmetries affected by these interactions. In this section we will concentrate on the cases where $L_{e_{R}}$ is violated by itself or in combination with soft electron lepton number violation.

\subsection{The evolution equations}

To derive the evolution equations for the $e_{R}$ and $e_{L}$ asymmetries we first write down the Boltzmann equations for the difference between the particle and antiparticle number densities. The collision terms in these equations correspond to the interactions shown in the table 1 and depicted in figure 1. It is reasonable to assume that each particle is in kinetic equilibrium at all times of interest; this is true in particular for the right-handed electrons due to their nonvanishing hypercharge. We then find

$$
\begin{aligned}
\left(\partial_{t}-3 H\right)\left(n_{e_{R}}-n_{e_{R}}\right)= & 2 \int \prod_{i=1}^{3} \mathrm{~d} \prod_{i}(2 \pi)^{4} \delta^{4}\left(p_{1}-p_{2}-p_{3}\right)\left|\mathcal{M}\left(h^{*} \leftrightarrow e_{L}^{c} e_{R}\right)\right|^{2} \\
& \quad \times\left\{f_{h^{*}}\left(1-f_{e_{L}}\right)\left(1-f_{e_{R}}\right)-f_{e_{L}} f_{e_{R}}\left(1+f_{h^{*}}\right)\right\}-\text { c.c. } \\
& +2 \int \prod_{i=1}^{4} \mathrm{~d} \prod_{i}(2 \pi)^{4} \delta^{4}\left(p_{1}+p_{2}-p_{2}-p_{3}\right) \sum_{f}\left\{\left|\mathcal{M}\left(f_{L}{ }^{c} f_{R} \leftrightarrow e_{L}{ }^{c} e_{R}\right)\right|^{2}\right. \\
& \quad \times\left[f_{f_{L}} f_{f_{R}}\left(1-f_{e_{L}}\right)\left(1-f_{e_{R}}\right)-f_{e_{L}} f_{e_{R}}\left(1-f_{f_{L}}\right)\left(1-f_{f_{R}}\right)\right] \\
& \quad+\cdots\}- \text { c.c. } \\
& \quad+\frac{1}{2}\left(\partial_{t}-3 H\right)\left(n_{e_{R}}-n_{e_{R}}\right) \\
& +\int \prod_{i=1}^{4} \mathrm{~d} \prod_{i}(2 \pi)^{4} \delta^{4}\left(p_{1}+p_{2}-p_{2}-p_{3}\right) \sum_{\ell=e, \mu, \tau}\left\{S_{\ell}\left|\mathcal{M}\left(h^{-} h^{-} \leftrightarrow \ell_{L} e_{L}\right)\right|^{2}\right.
\end{aligned}
$$




$$
\begin{aligned}
& \times\left[f_{h^{-}} f_{h^{-}}\left(1-f_{e_{L}}\right)\left(1-f_{\ell_{L}}\right)-f_{e_{L}} f_{\ell_{L}}\left(1+f_{h^{-}}\right)\left(1+f_{h^{-}}\right)\right] \\
& +\cdots\}- \text { c.c. }+\frac{1}{6}\left(\partial_{t}-3 H\right)\left(n_{B}-n_{B^{c}}\right)
\end{aligned}
$$

where $H$ is the Hubble expansion rate and we defined the usual phase space volume element as $\mathrm{d} \Pi \equiv \mathrm{d}^{3} p /(2 \pi)^{3} 2 E . f_{L}$ and $f_{R}$ are the chiral quarks or leptons. The symmetry factor $S_{\ell}$ in (6) is introduced to avoid double-counting in case of identical initial or final state particles: $S_{e}=\frac{1}{4}$ and $S_{\mu, \tau}=\frac{1}{2}$. According to our assumption of prevailing kinetic equilibrium, the distribution functions are the usual Fermi-Dirac or Bose-Einstein functions

$$
f_{i}\left(p, \mu_{i}\right)=\left(e^{\beta\left(E_{i}-\mu_{i}\right)} \pm 1\right)^{-1}
$$

where $\beta \equiv 1 / T$ and $T$ is the common temperature. The factors of 2 in front of the integrals in (6) come from summing over the equal contributions from both fields in each left handed doublet. This is evident because the couplings and therefore the temperature-dependent masses of the doublet components are identical. Similarly, the half in front of the first term in (7) arises because $e_{L}$-number is changed only in half of these interactions.

In eqs. (6-7) we explicitly wrote only a few representatives of the whole set of collision terms corresponding to the interactions listed in the table 1, the rest being denoted by ellipses. The c.c. terms that come from the evolution equation for $n_{f^{c}}$ are just the charge conjugates of the terms shown, and the term

$$
\frac{1}{6}\left(\partial_{t}-3 H\right)\left(n_{B}-n_{B^{c}}\right) \equiv \frac{1}{6} s \dot{B}
$$

in eq. (7) is due to the fast interactions of sphalerons, which change $e_{L}$ number at $1 / 6$ the rate at which they violate baryon number. (In eq. (9) we used the fact that $\dot{s} / s=-3 H$ in the adiabatically expanding universe.) To see that this term is necessary, recall that in the absence of the $\Delta L=2$ lepton-violating interaction, we have the conserved quantity $\frac{1}{3} B-L_{e}=\frac{1}{3} B-2 L_{e_{L}}-L_{e_{R}}$. If the $\Delta L=2$ scattering term in (7) is zero, we see that this conservation law will be satisfied only if the $\frac{1}{6} s \dot{B}$ term is present. Below we will express $B$ in terms of $L_{e_{R}}$ and $L_{e_{L}}$. Lastly, let us remark that the second term in (7) is particular to the model with electron lepton number violation and should be omitted when $\frac{1}{3} B-L_{e}$ is conserved. 
Equations (6-7) constitute a set of nonlinear equations for the chemical potentials that appear in the distribution functions $f_{i}\left(p, \mu_{i}\right)$. We can convert them into linear equations for the asymmetries by expanding the collision terms to first order in the $\beta \mu_{i}$, which are proportional to the asymmetries through eq. (5). Of course the resulting evolution equations are only valid for small chemical potentials, but this is a reasonable sacrifice because we are interested only in very small final asymmetries of the order of the present baryon asymmetry $\sim 10^{-10}$. Even in the case of a large initial asymmetry the nonlinearity would persist only for a very short time, after which the system would be well-described by the linear equations. Then performing the expansions in chemical potentials and using equations (1) to rewrite the chiral fermion asymmetries in terms of $\mu_{0}$, equations (6-7) become

$$
\begin{aligned}
\frac{\mathrm{d} L_{e_{R}}}{\mathrm{~d} t} & =-\frac{2 \beta}{s}\left(\mu_{e_{R}}-\mu_{e_{L}}+\mu_{0}\right) \times\left(I_{D}+I_{G}+\sum_{f} I_{f}\right) \\
\frac{\mathrm{d} L_{e_{L}}}{\mathrm{~d} t} & =-\frac{1}{2} \frac{\mathrm{d} L_{e_{R}}}{\mathrm{~d} t}-\frac{2 \beta}{s} \sum_{\ell=e, \mu, \tau}\left(\mu_{e_{L}}+\mu_{\ell_{L}}+2 \mu_{0}\right) I_{\Delta L, \ell}+\frac{1}{6} \dot{B}
\end{aligned}
$$

It should be noted that the chirality-changing part of the collision term in eq. (10) for $L_{e_{R}}$ is proportional to $\mu_{e_{R}}-\mu_{e_{L}}+\mu_{0}$, clearly showing that $L_{e_{R}}$ is driven towards the correct equilibrium value. These equations are not yet solvable because $\mu_{0}$ and the sphaleron term $\frac{1}{6} \dot{B}$ appearing on the r.h.s. of (10) must be written in terms of the asymmetries $L_{e_{R}}$ and $L_{e_{L}}$. We will return to this issue shortly.

The coefficients that multiply chemical potentials in (10) are defined as follows. The (inverse) decay process gives

$$
I_{D}=2 \int \prod_{i=1}^{3} \mathrm{~d} \Pi_{i}(2 \pi)^{4} \delta^{4}\left(p_{1}-p_{2}-p_{3}\right)\left|\mathcal{M}\left(e_{L}{ }^{c} e_{R} \leftrightarrow h^{*}\right)\right|^{2} f_{e 1}^{0} f_{e 2}^{0}\left(1+f_{h 3}^{0}\right) .
$$

Scatterings involving the gauge bosons ${ }^{4}$ contribute a term

$$
\begin{aligned}
I_{G}=2 \int & \prod_{i=1}^{4} \mathrm{~d} \Pi_{i}(2 \pi)^{4} \delta^{4}\left(p_{1}+p_{2}-p_{3}-p_{4}\right) \times \\
& \times\left\{\left|\mathcal{M}\left(e_{L}{ }^{c} e_{R} \leftrightarrow h^{*} A\right)\right|^{2} f_{e 1}^{0} f_{e 2}^{0}\left(1+f_{h 3}^{0}\right)\left(1+f_{A 4}^{0}\right)\right.
\end{aligned}
$$

\footnotetext{
${ }^{4}$ We thank Sonia Paban and Willy Fischler for originally pointing out this contribution to us.
} 


$$
\begin{aligned}
& +\quad\left|\mathcal{M}\left(A e_{R} \leftrightarrow h^{*} e_{L}\right)\right|^{2} f_{e 1}^{0} f_{A 2}^{0}\left(1-f_{e 3}^{0}\right)\left(1+f_{h 4}^{0}\right) \\
& \left.+\quad\left|\mathcal{M}\left(h e_{R} \leftrightarrow A e_{L}\right)\right|^{2} f_{e 1}^{0} f_{h 2}^{0}\left(1-f_{e 3}^{0}\right)\left(1+f_{A 4}^{0}\right)\right\},
\end{aligned}
$$

where $A$ refers to either of the vector bosons $W$ or $B$. The scatterings involving fermions give

$$
\begin{aligned}
& I_{f}=2 \int \prod_{i=1}^{4} \mathrm{~d} \Pi_{i}(2 \pi)^{4} \delta^{4}\left(p_{1}+p_{2}-p_{3}-p_{4}\right) \times \\
& \times\left.\left\{\mid \mathcal{M}\left(e_{L}^{c} e_{R}\right) \leftrightarrow f_{L}{ }^{c} f_{R}\right)\right|^{2} f_{e 1}^{0} f_{e 2}^{0}\left(1-f_{f 3}^{0}\right)\left(1-f_{f 4}^{0}\right)+ \\
&+\quad\left(\left|\mathcal{M}\left(f_{L} e_{R} \leftrightarrow f_{R} e_{L}\right)\right|^{2}+\left|\mathcal{M}\left(f_{R}{ }^{c} e_{R} \leftrightarrow f_{L}{ }^{c} e_{L}\right)\right|^{2}\right) \times \\
&\left.\quad \times\left[f_{e 1}^{0} f_{f 2}^{0}\left(1-f_{e 3}^{0}\right)\left(1-f_{f 4}^{0}\right)\right]\right\} .
\end{aligned}
$$

Finally, the lepton number violating collision term is

$$
\begin{aligned}
I_{\Delta L, \ell}=\int \prod_{i=1}^{4} \mathrm{~d} & \Pi_{i}(2 \pi)^{4} \delta^{4}\left(p_{1}+p_{2}-p_{3}-p_{4}\right) \times \\
\times \quad\{ & \left(S_{\ell}\left|\mathcal{M}\left(e_{L} \ell_{L} \leftrightarrow h^{-} h^{-}\right)\right|^{2}+\left|\mathcal{M}\left(e_{L} \nu_{\ell} \leftrightarrow h^{*} h^{-}\right)\right|^{2}\right) \times \\
\quad & \times f_{e 1}^{0} f_{\ell 2}^{0}\left(1+f_{h 3}^{0}\right)\left(1+f_{h 4}^{0}\right) \\
+ & \left(\left|\mathcal{M}\left(e_{L} h^{+} \rightarrow \ell_{L}{ }^{c} h^{-}\right)\right|^{2}+\left|\mathcal{M}\left(e_{L} h^{+} \rightarrow \nu_{\ell}{ }^{c} h^{*}\right)\right|^{2}+\left|\mathcal{M}\left(e_{L} h \rightarrow \nu_{\ell}{ }^{c} h^{-}\right)\right|^{2}\right) \times \\
& \left.\times f_{e 1}^{0} f_{h 2}^{0}\left(1-f_{\ell 3}^{0}\right)\left(1+f_{h 4}^{0}\right)\right\} .
\end{aligned}
$$

The distribution functions $f_{\alpha i}^{0}$ appearing in (11- 14) are the usual equilibrium functions defined in (8) for particle species $\alpha$ with zero chemical potential and momentum $p_{i}$.

There is a technical subtlety concerning the $s$-channel scattering contributions to the chirality-changing interactions. We have included the (inverse) decay contribution explicitly in our Boltzmann equations, which assumes that the Higgs particle is a thermalized member of the heat bath. However, the on-shell part of the Higgs exchange in the $s$-channel scattering physically corresponds to an inverse decay into a real Higgs boson followed immediately by a decay process. Thus the on-shell contribution in $s$-channel scattering introduces double counting of decay interactions and has to be subtracted away. Hence what we mean by the $s$-channel contributions in our Boltzmann equations is actually the on-shell subtracted 
part. We will discuss the subtraction procedure in section 3, along with the computations of matrix elements and thermal averages.

We still have the problem that in general $\mu_{0}$ is not simply related to $\mu_{e_{R}}$ and $\mu_{e_{L}}$. This would be the case for example if all the lepton numbers were violated by slow processes. Then four conditions would be needed to close the network of equations for the four softly-violated asymmetries [10]. But if we assume that only electron lepton number violation is slow, we can use the conservation laws for $\frac{1}{3} B-L_{\mu}$ and $\frac{1}{3} B-L_{\tau}$ (or the new chemical equilibrium conditions for $\mu_{\mu_{L}}$ and $\mu_{\tau_{L}}$ if these are violated) to express the remaining unknown chemical potentials in (10) in terms of $\mu_{e_{R}}$ and $\mu_{e_{L}}$. In particular we can parametrize $\mu_{0}$ as

$$
\mu_{0} \equiv a \mu_{e_{R}}+b \mu_{e_{L}}
$$

with $a$ and $b$ model-dependent constants, computable when the specific set of conservation laws and equilibrium conditions is given.

Using (2-3) and (15) one then finds

$$
B=\frac{1}{2}(1-13 a) L_{e_{R}}-\frac{1}{2}(1+13 b) L_{e_{L}} .
$$

By differentiating this with respect to time, we can write $\frac{1}{6} \dot{B}$ in terms of $\dot{L}_{e_{R}}$ and $\dot{L}_{e_{L}}$ in eqs. (10), so that they close to give

$$
\begin{aligned}
& \frac{\mathrm{d} L_{e_{R}}}{\mathrm{~d} t}=-\Gamma_{R L}\left((1+a) L_{e_{R}}-(1-b) L_{e_{L}}\right) \\
& \frac{\mathrm{d} L_{e_{L}}}{\mathrm{~d} t}=-\frac{5+13 a}{13(1+b)} \frac{\mathrm{d} L_{e_{R}}}{\mathrm{~d} t}-\sum_{\ell=e, \mu, \tau} \Gamma_{\Delta L, \ell}\left(a_{\ell} L_{e_{R}}+b_{\ell} L_{e_{L}}+c_{\ell}\right),
\end{aligned}
$$

where

$$
\begin{aligned}
\Gamma_{R L} & \equiv \frac{12}{T^{3}}\left(I_{D}+I_{G}+\sum_{f} I_{f}\right) ; \\
\Gamma_{\Delta L, \ell} & \equiv \frac{12}{T^{3}} I_{\Delta L, \ell} .
\end{aligned}
$$

In (17), the coefficients $a_{\ell}$ and $b_{\ell}$ are linear combinations of $a$ and $b$, and $c_{\ell}$ are related to the initial values of conserved quantities. For example, if there is fast violation of muon and 
tau lepton number, then $\mu_{\mu_{L}}=\mu_{\tau_{L}}=-\mu_{0}$, as will be shown in section 5 , and we obtain

$$
\begin{array}{cl}
a_{e}=\frac{24 a}{13(1+b)} & b_{e}=\frac{24}{13} \\
a_{\mu}=a_{\tau}=\frac{1}{2} a_{e} & b_{\mu}=b_{\tau}=\frac{1}{2} b_{e} \\
c_{i}=0 . &
\end{array}
$$

Nevertheless, in equations (17 18) the parameters $a, a_{i}, b$, and $b_{i}$ are left unspecified in order to stress the fact of their model-dependence, notably their dependence on whatever boundary conditions are imposed by the fast processes. Since these appear multiplying the rates, the temperature at which the asymmetries reach their asymptotic values can be accurately determined only after these parameters are known.

If there is no lepton number violation aside from sphaleron effects, that is if $\Gamma_{\Delta L}=0$, the equation for $\dot{L}_{e_{L}}$ in (17) is actually redundant. In that case one would use the conservation of $\frac{1}{3} B-L_{e}$ along with eq. (16) to express $L_{e_{L}}$ in terms of $L_{e_{R}}$ in the evolution equation for $\dot{L}_{e_{R}}$, which could then be solved for $L_{e_{R}}$ directly; this is exactly what we will do in section 4 below. If there does exist additional electron lepton number violation, $\frac{1}{3} B-L_{e}$ is not conserved and the more general form of (17) must be used.

\section{Rates for (inverse) decays and scatterings}

We are interested in the rate at which $e_{R}$ number violation occurs in the early universe, to determine at what temperature the associated interactions come into thermal equilibrium,

and in particular whether this can be close to the electroweak phase transition temperature. The dominant processes are usually the decays and inverse decays of Higgs bosons into $e_{R}$ and the lepton doublet $L_{e}$ shown in figure 1. As will be discussed in the next section, the answer to the question of whether an $e_{R}$ asymmetry relaxes completely before sphalerons go out of equilibrium at the electroweak phase transition, is exponentially sensitive to the total rate of $e_{R}$ violation. We have therefore undertaken to more carefully estimate this rate than was done in CDEO. We will find that for very small Higgs boson masses $m_{H}$, inverse decays by themsleves are slow enough so that $e_{R}$ violation would not take place until after sphalerons 
had largely disappeared, in which case the baryon asymmetry would survive despite the vanishing of $B-L$. But before making this conclusion one should consider corrections to the rate of $e_{R}$ violation due to two-body scattering processes. It will be shown that these actually dominate over the decays for small $m_{H}$, leading to a large enough rate of $e_{R}$ violation that the results of CDEO are qualitatively unchanged.

\subsection{Decays}

For the (inverse) decay rate, we will make several refinements on the treatment given by CDEO: inclusion of the thermal masses of the leptons, use of the finite-temperature wave functions for the fermions, and retention of the Bose-Einstein statistical factor for the Higgs boson. The first two corrections are important in the limit of small $m_{H}$ because they exacerbate the the phase space suppression for the decay.

The decay rate entering into the Boltzmann equation (10) is proportional to an integral $I_{D}$, eq. (11), in which appears the transition matrix element $\mathcal{M}$. In terms of the electron Yukawa coupling $h_{e}$, the squared matrix element for $H \rightarrow e_{R} e_{L}^{c}$ is

$$
|\mathcal{M}|^{2}=2 h_{e}^{2} \hat{p}_{e_{L}} \cdot \hat{p}_{e_{R}}=2 h_{e}^{2}\left(\left|\vec{p}_{e_{L}}\right|\left|\vec{p}_{e_{R}}\right|-\vec{p}_{e_{L}} \cdot \vec{p}_{e_{R}}\right)
$$

where $\hat{p}_{e_{L}}$ and $\hat{p}_{e_{R}}$ are light-like four-momenta constructed from the corresponding threemomenta. These appear in place of the usual four-momenta because the fermion masses are from thermal effects which break Lorentz invariance but preserve chirality, so that one must still use massless spinors in constructing the amplitude [11]. However to a good approximation the dispersion relations for the particles appear to be massive. The thermal masses of the two chiralities of electrons and the Higgs, appropriate to the temperatures above the electroweak phase transition in which we are interested, are given by ${ }^{5}$ [11, 12]

$$
\begin{aligned}
& m_{e_{L}}^{2}(T) / T^{2}=\left(3 g^{2}+g^{\prime 2}\right) / 32 \cong 0.044 \\
& m_{e_{R}}^{2}(T) / T^{2}=g^{\prime 2} / 8 \cong 0.017
\end{aligned}
$$

\footnotetext{
${ }^{5}$ We note that the coefficient of $m_{0}^{2}$ in the expression for $m_{H}^{2}(T)$ is corrected here relative to that which was quoted in ref. 8 .
} 


$$
\begin{aligned}
m_{H}^{2}(T) / T^{2} & =2 D\left(1-T_{c}^{2} / T^{2}\right) \\
D & \equiv\left(2 m_{W}^{2}+m_{Z}^{2}+2 m_{t}^{2}+m_{0}^{2}\right) / 8 v^{2} .
\end{aligned}
$$

Here $m_{0}$ is the zero-temperature Higgs boson mass, and $g$ and $g^{\prime}$ are the respective $\mathrm{SU}(2)_{L}$ and $\mathrm{U}(1)_{Y}$ couplings evaluated at the running scale of $100 \mathrm{GeV}$ which is at the lower limit of the temperatures we are interested in. $T_{c}$ is the critical temperature for the electroweak transition, given at one loop by

$$
T_{c}^{2}=\frac{1}{4 D}\left(m_{H}^{2}-\frac{3}{8 \pi^{2} v^{2}}\left(2 m_{W}^{4}+m_{z}^{4}-4 m_{t}^{4}\right)-\frac{1}{8 \pi^{2} v^{4} D}\left(2 m_{W}^{3}+m_{Z}^{3}\right)^{2}\right) .
$$

By substituting the lowest experimentally allowable values of the top quark mass $m_{t}=90$ $\mathrm{GeV}$ and $m_{0}=60 \mathrm{GeV}$, we find that $m_{H} / T$ must be no smaller than 0.41 in the high temperature limit, where it becomes constant as a function of temperature. This also approximately coincides with the kinematic threshold for thermal Higgs boson decays into electrons. $m_{H} / T$ is the key parameter for the strength of the decays because $I_{D}$ depends entirely upon it, considering $m_{e_{L}}$ and $m_{e_{R}}$ to be known quantities. In Figure 2 we have plotted the thermal Higgs mass for various values of $m_{0}$ and $m_{t}$, where it can be seen that $m_{H}$ varies between 0.4 and 2 for $m_{0}$ between 60 and $1000 \mathrm{GeV}$, for relevant values of the top quark mass.

The integral in eq. (11), which is proportional to the thermally averaged rate of decays, can be reduced to one dimension:

$$
I_{D}=\frac{m_{H} T^{3} h_{e}^{2} \gamma^{2}}{\pi^{3}} \int_{1}^{\infty} \mathrm{d} u \frac{e^{u m_{H} / T}}{\left(e^{u m_{H} / T}-1\right)^{2}} \ln \left(\frac{\cosh \left(\alpha_{e_{L}} u+\gamma \sqrt{u^{2}-1}\right)}{\cosh \left(\alpha_{e_{L}} u-\gamma \sqrt{u^{2}-1}\right)} \frac{\cosh \left(\alpha_{e_{R}} u+\gamma \sqrt{u^{2}-1}\right)}{\cosh \left(\alpha_{e_{R}} u-\gamma \sqrt{u^{2}-1}\right)}\right),
$$

where

$$
\begin{aligned}
\alpha_{e_{L}} & \equiv\left(m_{H}^{2}+m_{e_{L}}^{2}-m_{e_{R}}^{2}\right) / 4 m_{H} T ; \\
\alpha_{e_{R}} & \equiv\left(m_{H}^{2}+m_{e_{R}}^{2}-m_{e_{L}}^{2}\right) / 4 m_{H} T ; \\
\gamma & \equiv \lambda^{1 / 2}\left(m_{H}^{2}, m_{e_{L}}^{2}, m_{e_{R}}^{2}\right) / 4 m_{H} T ; \\
\lambda(x, y, z) & \equiv(x-y-z)^{2}-4 y z .
\end{aligned}
$$

In CDEO, $I_{D}$ was approximated by $\left(h_{e} m_{H} T \ln 2\right)^{2} /\left(16 \pi^{3}\right)$. In Table 2 we compare the numerically integrated value with this approximation for some values of $m_{H} / T$. 


\begin{tabular}{|c|c|c|c|c|}
\hline \hline$m_{H} / T$ & $I_{D, C D E O} / K$ & $I_{D} / K$ & $I_{t o p} / K$ & $I_{G} / K$ \\
\hline 0.4 & $1.6 \times 10^{-4}$ & $2.5 \times 10^{-5}$ & $4.2 \times 10^{-5}$ & $3.2 \times 10^{-4}$ \\
0.6 & $3.5 \times 10^{-4}$ & $2.0 \times 10^{-4}$ & $4.2 \times 10^{-5}$ & $"$ \\
0.8 & $6.2 \times 10^{-4}$ & $4.1 \times 10^{-4}$ & $4.1 \times 10^{-5}$ & $"$ \\
1.0 & $1.0 \times 10^{-3}$ & $6.4 \times 10^{-4}$ & $4.0 \times 10^{-5}$ & $"$ \\
1.2 & $1.4 \times 10^{-3}$ & $8.5 \times 10^{-4}$ & $3.7 \times 10^{-5}$ & $"$ \\
1.4 & $1.9 \times 10^{-3}$ & $1.1 \times 10^{-3}$ & $3.4 \times 10^{-5}$ & $"$ \\
1.6 & $2.5 \times 10^{-3}$ & $1.2 \times 10^{-3}$ & $3.2 \times 10^{-5}$ & $"$ \\
1.8 & $3.1 \times 10^{-3}$ & $1.4 \times 10^{-3}$ & $2.9 \times 10^{-5}$ & $"$ \\
2.0 & $3.9 \times 10^{-3}$ & $1.5 \times 10^{-3}$ & $2.7 \times 10^{-5}$ & $"$ \\
\hline \hline
\end{tabular}

Table 2: The quantities $I_{a}$ that appear in the Boltzmann equation, divided by $K=h_{e}^{2} T^{4}$. The subscript CDEO refers to the approximations for the decay rate made in reference [8], while $D$, top, and $G$ refer respectively to the exact decay rate and the scattering rates for top quarks and gauge bosons to produce $e_{R}$.

\subsection{Scatterings producing $e_{R}$}

One might expect that two-body scatterings which change $e_{R}$ number are slower than decays because of phase space and coupling constant suppression. However in the region of small thermal Higgs boson masses the phase space for the decay is strongly suppressed. In this regime the production of electrons by top quark scattering (Figure 3) or Higgs/gauge boson scattering (Figure 4) may actually dominate over the decays.

In the Boltzmann equation, the thermally averaged scattering rates for processes which change $e_{R}$ number appear through the integrals $I_{f}$ and $I_{G}$ defined in eqs. (12 13). We may ignore all the fermion scattering processes except for $f=$ top since the top quark has the dominant Yukawa coupling. The channels which produce an $e_{R}$ in the final state are listed in Table 1, and are categorized here as top-quark or gauge/Higgs boson scatterings. We use the Maxwell-Boltzmann approximation for the initial state particles, and neglect the distribution functions compared to unity for the final state particles. The errors introduced by this procedure are irrelevant in the regime of large $m_{H} / T$ where the scatterings are small corrections to the decay rate; in the small $m_{H} / T$ regime, although the scatterings dominate, 
they are still sufficiently fast for our purposes that the anticipated $20 \%$ corrections to the Maxwell-Boltzmann approximation would not change any of our subsequent conclusions. Then the quantities $I_{i}$ can be expressed as an integral over the Mandelstam variable $s$ involving the corresponding cross sections and the modified Bessel function $K_{1}$ :

$$
I_{i}=\frac{T}{32 \pi^{4}} \sum_{c} \int_{M_{c}^{2}}^{\infty} \frac{d s}{s^{1 / 2}} \lambda\left(s, m_{1}^{2}, m_{2}^{2}\right) K_{1}(\sqrt{s} / T) \sigma_{c}(s)
$$

The sum is over all possible channels $c$, each of which has a threshold determined by the thermal masses of the initial or final state particles, $M_{c}=\max \left(m_{1}+m_{2}, m_{3}+m_{4}\right)$. The sum includes a factor of 2 for isospin (since all the processes we consider involve doublets) and if there are gauge bosons, a factor of 3 for the polarizations. Note that there is no corresponding factor for the fermion spins because they all have a definite helicity. The sum also runs over the different crossings that leave $e_{R}$ in the final state. The choice of whether $e_{R}$ is in the initial or final state is arbitrary-either way gives the same final answer.

In performing the thermal averages of the cross sections, we omitted corrections of order $m^{2} / s$, where $m$ is a thermal mass, since typically $m<T$ and $s \sim 20 T^{2}$. Thus we can set the threshold to zero in the integration of eq. (25) and approximate $\lambda\left(s, m_{1}^{2}, m_{2}^{2}\right)$ by $s^{2}$. (The same neglect of thermal masses would not have been appropriate for accurately determining the decay rate since the phase space there is determined by the scale $m_{H}$ rather than $s$.) In this approximation, the analytic expressions for the top quark scattering cross sections are

$$
\sum_{c} \sigma_{c}(s)=\frac{\left(h_{e} h_{t}\right)^{2}}{8 \pi s}\left(\frac{s^{2}}{\left(s-m_{H}^{2}\right)^{2}+s \Gamma^{2}}+2\right)
$$

and for gauge/Higgs boson scattering

$$
\sum_{c} \sigma_{c}(s)=\frac{4 h_{e}^{2}}{\pi s T^{2}}\left(m_{e_{R}}^{2} \ln \frac{s}{m_{e_{R}}^{2}}+m_{e_{L}}^{2} \ln \frac{s}{m_{e_{L}}^{2}}-\frac{5}{4} m_{e_{L}}^{2}-\frac{7}{4} m_{e_{R}}^{2}\right),
$$

where the thermal masses of eq. (21) have been used and $\Gamma$ is the width of a Higgs boson with mass $\sqrt{s}: \Gamma(s) \cong \frac{h_{t}^{2}}{16 \pi} \sqrt{s}$. Some care must be taken to obtain the subleading terms correctly. The numerical values of the above contributions to the Boltzmann equation for the $e_{R}$ asymmetry are also shown in Table 2. We have assumed a top quark Yukawa coupling 
corresponding to a mass of $90 \mathrm{GeV}$ in computing $I_{t o p}$. Although this may be unrealistically small, we wanted to examine the regime of small $m_{H} / T$ which is most favorable to the possibility of keeping $e_{R}$-changing interactions out of equilibrium below the freeze-out of sphalerons, and eq. (21) shows that this occurs for the smallest values of $m_{t}$ and $m_{0}$. However, Table 2 shows that the contributions from scattering dominate those of decays in the low- $m_{H}$ region, so that one cannot in fact evade the washout of an $e_{R}$ asymmetry even by going to small masses. More will be said about this in section 4 .

\subsection{Subtraction of Resonances}

As has been mentioned above, the $s$-channel scattering $t_{R} t_{L}^{c} \rightarrow e_{R} e_{L}^{c}$ contains an on-shell part which must be subtracted to avoid double-counting the contribution from the inverse decays. This necessity has been mentioned in references [13] and [14], although the details of how to carry it out were not explained. We have considered two methods that seem reasonable and are in good quantitative agreement with each other. Writing the finitewidth, squared $s$-channel propagator for the Higgs as $P(s ; \Gamma)=\left(\left(s-m_{H}^{2}\right)^{s}+s \Gamma^{2}\right)^{-1}$, we can summarize the different methods by making the following modifications to the propagator, which we refer to respectively as the principal value (PV) and delta function subtraction (SDF) methods:

$$
\begin{array}{cl}
\mathrm{PV}: & P(s ; \Gamma) \rightarrow P(s+i \epsilon ; 0) ; \\
\mathrm{SDF}: & P(s ; \Gamma) \rightarrow P(s ; \Gamma)-\frac{\pi}{\sqrt{s} \Gamma} \delta\left(s-m_{H}^{2}\right) ;
\end{array}
$$

Note that the PV method gives $\int_{-a}^{b} x^{-2} d x=-a^{-1}-b^{-1}$, even when the double pole is straddled by $-a$ and $b$. The SDF method attempts to isolate the off-shell part by subtracting what the scattering rate "would have been" if the resonance were infinitesimally narrow. Although these alternatives at first look quite different from each other, by writing $\pi \delta\left(s-m_{H}^{2}\right)$ as $\epsilon /\left(\left(s-m_{H}^{2}\right)^{2}+\epsilon^{2}\right)$ one can show that they are actually equal to each other in the limit as $\Gamma \rightarrow 0$; writing $x=\left(s-m_{H}^{2}\right)$, and $\epsilon^{\prime}=\epsilon \Gamma$, the difference is

$$
\frac{x^{2}-\epsilon^{\prime 2}}{\left(x^{2}+\epsilon^{\prime 2}\right)^{2}} \text { versus } \frac{x^{2}-\epsilon^{\prime 2}}{\left(x^{2}+\epsilon^{\prime 2}\right)^{2}+x^{2} \Gamma^{2}} \text {. }
$$




\begin{tabular}{|c|r|r|}
\hline \hline$m_{H} / T$ & \multicolumn{1}{c|}{$\mathrm{PV}$} & \multicolumn{1}{c|}{$\mathrm{SDF}$} \\
\hline 0.4 & $1.52 \times 10^{-5}$ & $1.52 \times 10^{-5}$ \\
0.8 & $1.43 \times 10^{-5}$ & $1.42 \times 10^{-5}$ \\
1.2 & $1.04 \times 10^{-5}$ & $1.04 \times 10^{-5}$ \\
1.6 & $5.24 \times 10^{-6}$ & $5.33 \times 10^{-6}$ \\
2.0 & $3.95 \times 10^{-7}$ & $3.58 \times 10^{-7}$ \\
2.4 & $-3.53 \times 10^{-6}$ & $-3.53 \times 10^{-6}$ \\
2.8 & $-6.15 \times 10^{-6}$ & $-6.16 \times 10^{-6}$ \\
3.2 & $-7.14 \times 10^{-6}$ & $-7.55 \times 10^{-6}$ \\
3.6 & $-7.70 \times 10^{-6}$ & $-7.67 \times 10^{-6}$ \\
\hline \hline
\end{tabular}

Table 3: Contributions to $I_{\text {top }} / K$ (see table 2) due to $s$-channel Higgs boson scattering. We compare the principal value prescription used in table 2 to the delta function subtraction method.

In fact this is as good a correspondence as one should hope for since the whole concept of asymptotic states which have thermal distributions breaks down if they become very broad resonances. Thus two subtraction schemes should be considered equally good if they differ only by terms of order $\Gamma^{2}$. We compare the PV and SDF methods in Table 3, of which the former was used in the top-quark contributions shown in Table 2.

The negative values for $m_{H} / T>2.0$ may be alarming at first, since they are supposed to be the off-shell part of a scattering rate. Although our results are completely insensitive to the negative contributions because of their relative smallness compared to the decay rate, it is interesting to speculate on their meaning. We take them as being suggestive that the decay rate $I_{D}$ gives an overestimate of the Higgs-mediated processes at large values of $m_{H} / T$. In this regime, due to strong couplings to itself or the top quark, the Higgs boson is starting to become a rather broad resonance where it may not be sensible to consider the decays; rather one should include the scatterings only, without subtracting the resonance from the $s$-channel. These give a smaller contribution than the decays because the area under the resonance curve gets diminished by the Boltzmann suppression in the function $K_{1}(\sqrt{s} / T) \sim \exp (-\sqrt{s} / T)$ (eq. (25)). But since the off-shell scatterings are completely 
negligible compared to the decays in the large $m_{H} / T$ regime, the question of whether to take the negative cross-section seriously is moot-its contribution is negligible.

\section{4 $\Delta L=2$ Scatterings}

In a later section we will also be interested in how $\Delta L=2$ lepton number violating interactions come into equilibrium, in connection with baryon number erasure. The operator we consider, which gives rise to neutrino masses, has dimension five:

$$
\sum_{i j} \frac{m_{i j}}{2 v^{2}}\left(\bar{L}_{i} H\right)\left(1+\gamma_{5}\right)\left(H^{T} L_{j}^{c}\right)+\text { h.c. }
$$

where $m_{i j}$ is the neutrino mass matrix in the flavor basis, $v$ is the $246 \mathrm{GeV}$ VEV of the Higgs field $H$, and $L_{i}$ are the lepton doublets.

The sum over channels of the cross sections for the processes $e_{L} \nu_{\ell} \rightarrow h^{*} h^{-}$and $e_{L} \ell_{i} \rightarrow$ $h^{-} h^{-}$must be properly weighted by the factors $S_{\ell}$ shown in the Boltzmann equation (7) for $e_{L}$, because of the complication of having identical particles in some of the initial or final states. We find that

$$
\sum_{c} \sigma_{c, \ell}(s)=\frac{1}{4 \pi v^{4}} \begin{cases}5\left|m_{e e}\right|^{2}, & \ell=e \\ 6\left|m_{e \ell}\right|^{2}, & \ell=\mu, \tau .\end{cases}
$$

This is what we must substitute into eq. (25) to obtain the quantities $I_{\Delta L, \ell}$, which appear in eq. (21) for the rate of lepton violation. The various channels respectively contribute the relative weights $(2,2,4,1,1)\left|m_{e e}\right|^{2}$ for $e_{L} e_{L} \rightarrow h^{-} h^{-}, e_{L} \nu_{e} \rightarrow h^{-} h^{*}, e_{L} h^{+} \rightarrow e_{L}^{c} h^{-}, e_{L} h^{+} \rightarrow$ $\nu_{e}{ }^{c} h^{*}, e_{L} h \rightarrow \nu_{e}^{c} h^{-}$and $(4,2,4,1,1)\left|m_{e \ell}\right|^{2}$ for $e_{L} \ell_{L} \rightarrow h^{-} h^{-}, e_{L} \nu_{\ell} \rightarrow h^{-} h^{*}, e_{L} h^{+} \rightarrow \ell_{L}^{c} h^{-}$, $e_{L} h^{+} \rightarrow \nu_{\ell}^{c} h^{*}, e_{L} h \rightarrow \nu_{\ell}^{c} h^{-}$with $\ell=\mu, \tau$. In the approximation of ignoring masses compared to $s$, the integral in eq. (25) can be done exactly,

$$
I_{\Delta L, \ell}=\frac{T^{6}}{\pi^{4}} \sum_{c} \sigma_{c, \ell} .
$$

This simplification occurs because of the constant cross section. Of course at very high temperatures of order the mass $M$ of the heavy neutrino which must have induced the operator (30), the cross section will exhibit a form factor behaving as $M^{2} /\left(T^{2}+M^{2}\right)$, coming from the propagator. Thus we are assuming that $M$ is greater than the temperatures of 
interest when we use eq. (32). It will be shown later that as long as $M$ exceeds $10 \mathrm{TeV}$, our neglect of the propagator is justified.

\section{The case of conserved $B-L$}

The importance of the weak electron chirality-changing interactons in the context of preserving the baryon asymmetry was first discussed by the authors of ref. [8] (CDEO). They considered whether in a universe with primordial $B-L=0$, the right-handed electrons could stay out of equilibrium long enough so that the primordial baryon asymmetry, kept nonzero by the asymmetry in the $e_{R}$ species, does not get washed out completely before the sphaleron interactions fall out of equilibrium. While their result was negative for the preservation of the baryon asymmetry, they noted that the outcome of the computation is exponentially sensitive to the strength of the chirality-changing interactions. Therefore even a rather small change in the interaction rates could alter the conclusion.

Indeed, the derivation of CDEO contained a number of inaccuracies with the potential to change the outcome significantly. In particular, their neglect of the final state particle masses

leads to an overestimate of the rate of Higgs decays, and therefore to an underestimate of the final asymmetry. As we have seen in the section 3 above, the finite mass effects are very important for small (thermal) Higgs boson masses. On the other hand, CDEO neglected all scattering processes, which we found to be of particular importantance when the decay process is suppressed by the phase space effects. Armed with our accurate expressions for all tree level chirality changing rates computed in the previous section, as well as with the carefully formulated evolution equations derived in section 2, we can now re-examine and answer definitively the question CDEO posed.

As explained in section 3, the evolution equations for the asymmetries depend on the particular set of equilibrium conditions and conservation laws characterizing the system. Here the set of boundary conditions is given by the equilibrium equations (11 3 ) supplemented with the additional constraint of vanishing $B-L$. In fact we only assume that primordially $(B-L)_{p}=0$; the vanishing of $B-L$ at any lower temperature is guaranteed by the first three of the conservation laws (丑), which also imply the conservation of $B-L$. In terms of 
the chemical potentials we then find the constraint

$$
B-L=12 \mu_{u_{L}}-3 \sum_{\ell} \mu_{\ell_{L}}-2 \mu_{e_{L}}-\mu_{e_{R}}+2 \mu_{0}=0 .
$$

From eqs. (2-3) and (33) one can readily find that

$$
\begin{aligned}
\mu_{0} & =\frac{5}{153}\left(\mu_{e_{R}}-\mu_{e_{L}}\right) \\
\mu_{B} & =\frac{44}{153}\left(\mu_{e_{R}}-\mu_{e_{L}}\right) .
\end{aligned}
$$

Note that if we further assumed the $e_{R}$ population is in equilibrium, i.e. $\mu_{e_{R}}-\mu_{e_{L}}=-\mu_{0}$, then only the trivial solution would exist: $\mu_{0}=B=L=0$, in agreement with the standard analysis of ref. [4].

Our goal is to follow the evolution of the $e_{R}$ asymmetry using eq. (10), where from (34) we see that the constants $a, b$ are given by $a=-b=5 / 153$. However, we need not consider coupled equations for $L_{e_{L}}$ and $L_{e_{R}}$ because the latter is determined through the conservation law $\frac{1}{3} B-L_{e}=C_{e, p}$. Eliminating $B$ from this equation using (34) for $\mu_{B} \propto B$, we find that

$$
L_{e_{L}}=-\frac{415}{962} L_{e_{R}}-\frac{459}{962} C_{e, p}
$$

Inserting this into eq. (10) then gives the simple first order equation for $L_{e_{R}}$

$$
\frac{\mathrm{d} L_{e_{R}}}{\mathrm{~d} t}=-\Gamma\left(L_{e_{R}}+\frac{1}{3} C_{e, p}\right) .
$$

where

$$
\Gamma=\frac{711}{481} \Gamma_{R L} \cong \frac{17.7}{T^{3}}\left(I_{D}+I_{G}+I_{t o p}\right) .
$$

The quantities $I_{x} / T^{3}$ are the thermally averaged rates computed in section 2. Eq. (36) is easily integrated to yield a general solution for $L_{e_{R}}$ as a function of time that is completely specified in terms of primordial quantities:

$$
L_{e_{R}}(t)=-\frac{1}{3} C_{e, p}+\left(L_{e_{R}, p}+\frac{1}{3} C_{e, p}\right) e^{-\int_{t_{0}}^{t} \mathrm{~d} t^{\prime} \Gamma} .
$$

Interestingly, the right handed electron asymmetry does not tend to zero, but instead relaxes to a fixed value $-\frac{1}{3} C_{e, p}$ which may be even larger in absolute value than the primordial 
value of $L_{e_{R}}$. This is contrary to the assertion of CDEO that $L_{e_{R}} \rightarrow 0$. What happens is that at the fixed point the right and left chiral asymmetries become equal under the additional constraint of $\left(\frac{1}{3} B-L_{e}\right)$-conservation. This results in erasing the baryon and lepton asymmetries, $B, L \rightarrow 0$, as can be inferred from eq. 34. It is useful to write down an expression for $B$ directly as a function of time

$$
B(t)=B_{e q} e^{-\int_{t_{0}}^{t} \mathrm{~d} t^{\prime} \Gamma}
$$

where the equilibrium value of $B$ much above $T_{*}$ is given by

$$
B_{e q}=\frac{66}{481}\left(C_{e, p}+3 L_{e_{R}, p}\right) .
$$

As expected, the value of $B$ decreases exponentially in time, simultaneously with the equilibration of the $e_{R}$ population. It is conceivable that the initial value for $B_{e q}$ could be as large as $B^{e q} \sim 10^{-2}$ in some grand unified theories, although typically it will be suppressed by small coupling constants and $C P$-violating phases. We will allow $B^{e q}$ to vary in what follows.

Equation (39) is our main result in this section. Although we will compute the rate $\Gamma$ and its time integral for (39) numerically, to get a feeling for the relevant scales it is useful to find an analytic approximation for $B_{\text {final }}$. We can do this by employing linear fits to the values of the functions $I_{x}$ given in table 2, and integrating the ensuing fit functions analytically. Using a simple linear regression formula, we find

$$
\Gamma_{R L} \simeq 3.8 \times 10^{-3} T h_{e}^{2} f\left(m_{H}(T) / T\right),
$$

where the fit function

$$
f(x) \equiv(-1.1+3.0 x)+1.0+h_{t}^{2}(0.6-0.1 x)
$$

has been normalized so that the middle term, due to gauge/Higgs scattering, is unity. Because the number of degrees of freedom is constant, $g_{*}=106.75$, entropy is conserved and we can use the approximation $\dot{T} / T=-H$. Then, neglecting the threshold effect in the decays, we obtain the result

$$
\int_{0}^{t_{c}} \mathrm{~d} t \Gamma \simeq 350\left(\frac{100 \mathrm{GeV}}{T_{c}}\right)\left[\left(-1.1+2.4 x_{H}\right)+1.0+h_{t}^{2}\left(0.6-0.09 x_{H}\right)\right],
$$


where we have defined the asymptotic high-temperature limit of the Higgs boson thermal mass as

$$
\lim _{T \rightarrow \infty} m_{H}(T) / T=\sqrt{2 D} \equiv x_{H}
$$

The approximation (43) treats the decay part too roughly for small thermal Higgs masses, and therefore cannot be used to obtain figure 4a. However it is a very good estimate for the total integrated rate over the whole interesting range of $x_{H} \lesssim 2$. It should be noted that for a large range of thermal Higgs masses, $x_{H} \lesssim 0.9$, the scattering contribution to the integrated rate dominates over the decay part.

In figure 5 we show the result of our computation for the final baryon asymmetry in units of the initial asymmetry (40), as a function of the physical Higgs and top quark masses. For comparision to the work of CDEO, we show in figure 5a what the outcome would have been, had we included only the corrected decay contribution to the total rate. The curves are contours of constant $B_{\text {final }}$. The initial upward bending of the curves as a function of the zero-temperature Higgs boson mass $m_{0}$ occurs because the endpoint of the integration, the electroweak phase transition temperature $T_{c}$, increases with $m_{0}$. The resulting decrease in the integrated rate can be compensated by increasing $m_{t}$. As $m_{0}$ continues to increase, the thermal Higgs mass starts to show its dependence on $m_{0}$ (cf. eq. 21) leading to a significant increase in the rate as a function of $m_{0}$. Simultaneously the phase transition temperature reaches a plateau and therefore the contours turn down at large values of $m_{0}$.

It is noteworthy, and in contrast to the result of CDEO, that their mechanism could have appeared to be successful in protecting the baryon asymmetry for a small region of experimentally allowed parameter space, had they considered only the more exact expression for the Higgs boson decay rate. The main reason for this would-be positive result is the suppression of the decay rate for small values of $x_{H}$, where the phase space volume is small. When the scattering contributions are included however, the conclusion changes drastically. We show the result of the numerical integration in figure 5b. In particular, because of the gauge scatterings the final baryon asymmetry is completely negligible over the entire parameter space. Thus the scatterings play a decisive role in the most relevant region of parameters for the problem. In the regime where the decay contribution dominates over the 
scattering rate, $B_{\text {final }}$ is even more suppressed than in the region of parameters shown in figure $5 \mathrm{~b}$, which extends only up to $x_{H} \sim 0.55$.

Our conclusion is qualitatively the same as that of CDEO: it is not possible to protect the baryon asymmetry of a $B-L=0$ universe by temporarily storing it in the asymmetry of the $e_{R}$ species. However, the way in which we reached this conclusion differed from theirs in important respects and moreover, the robustness of our result erases any doubts expressed by CDEO, that some subtle thermal effects might alter the outcome.

\section{Examples when $B-L$ is not conserved}

Although we have seen that an $e_{R}$ asymmetry cannot save the baryon asymmetry from sphaleron effects when $B-L=0$, it can have a dramatic effect when $B-L \neq 0$, as one might have in $\mathrm{SO}(10)$ grand unified theories where $B-L$ gets broken at the GUT scale. In this section we will explore the consequences of an $e_{R}$ asymmetry in conjunction with sphalerons and other $B$ - and $L$-violating interactions that hitherto were believed to completely erase $B$.

It is expected that any global symmetries in nature are only approximate; they might be broken by exotic gravitational means such as microscopic black holes or wormholes, or more mundane reasons like the fact that one can construct renormalizable $B$ - and $L$-violating interactions in supersymmetry using the squark and slepton fields. Such effects may be described by nonrenormalizable operators in the low energy effective theory. Assuming that a primordial baryon asymmetry survives allows us to constrain the strength of these new interactions. These constraints were first applied to the $\Delta L=2$ operator (30) by Fukugita and Yanagida [3]. They were generalized to other operators in refs. [5, 6].

Quite generally, an operator of dimension $4+n$ would have a dimensionful coefficient of the form $M^{-n}$, and would mediate a process at high temperatures whose rate scales like $\Gamma \sim T^{2 n+1} M^{-2 n}$. This rate is to be compared to the Hubble expansion rate $\sim T^{2} / M_{P l}$. Insisting that the interaction be out of equilibrium below the temperature $T_{0}$ at which sphalerons came into equilibrium $\left(10^{12} \mathrm{GeV}\right)$, or when the baryon asymmetry was formed, 
whichever was smaller, leads to a lower bound on the scale of new physics:

$$
M \gtrsim T_{0}\left(\frac{M_{P l}}{T_{0}}\right)^{1 / 2 n}
$$

In supersymmetric models it was argued 15 that due to additional anomalies which can temporarily protect the asymmetry (until the effects of supersymmetry breaking kick in), the maximum temperature should be at $T_{0} \sim 10^{8} \mathrm{GeV}$ rather than $T_{0} \sim 10^{12} \mathrm{GeV}$. Interestingly, in the context of inflation the maximum temperature should not surpass the thermalization temperature [16], which could be as low as $\sim 10^{5} \mathrm{GeV}$ [17]. We will argue that actually none of these values of $T_{0}$ are the correct temperature to use in setting this bound whenever an $e_{R}$ asymmetry is present, as it will prevent the baryon asymmetry from relaxing to zero, essentially because of the charge neutrality of the universe. Rather, one should use the temperature $T_{*}$ at which $e_{R}$-violation comes into equilibrium and allows the $e_{R}$ asymmetry to disappear. In this section we will compute $T_{*} \simeq 1 \mathrm{TeV}$. Hence constraints like (45) might be weakened by as many as nine orders of magnitude, by standard model physics which has been known for well over a decade! Note, however, that our arguments here do not affect the limits imposed on renormalizable $B$ - or $L$-violating operators. For these, the strongest bound always comes from applying the limit at $T_{c}$ (when sphalerons disappear) [5].

Before computing $T_{*}$, we will follow the evolution of the baryon and $e_{R}$ asymmetries more exactly, for a particular example of a nonrenormalizable operator, that which would be responsible for the electron neutrino mass. An example employing explicit $B$-violation will also be given.

\section{$5.1 \quad$ Neutrino masses}

The best known example of $B-L$ violation is the possibility of neutrino Majorana masses, which would be generated by the dimension five operator, $\sum m_{i j} v^{-2}\left(L_{i} H\right)\left(L_{j} H\right)$. To avoid an erasure of the baryon asymmetry, upper limits on $m_{i j}$, the neutrino mass matrix elements, must be satisfied [3, 4, 18]. The flavor-dependence of the operator will be important since any approximately conservered quantities (such as $\frac{1}{3} B-L_{i}$ ) would protect the asymmetry [18, 19]. If for all flavors the operator was so weak as never to have been in equilibrium, and 
$B-L=0$, then as shown in the previous section, the baryon asymmetry would be erased, up to small mass effects [20, 19]. If, on the other hand, one or two of the lepton generations were violated by this operator and the remaining generations were out of equilibrium, then even if $B=L=0$ (or more generally, $B-L=0$ ) a baryon number would be generated provided some initial lepton flavor asymmetry existed [8]. But if all three flavors were violated down to low enough temperatures, the baryon asymmetry would be erased. How low is low enough? The answer, which we will denote by $T_{*}$, depends crucially on the decoupling temperature of the chirality-flipping electron interactions. In this section we will determine $T_{*}$, as well as the size of the neutrino mass matrix elements which would be compatible with preserving a primordial baryon asymmetry.

Supposing that the masses of $\nu_{\mu}$ and $\nu_{\tau}$ were sufficiently large (several $\mathrm{keV}$ ) that the separate lepton flavors $L_{\mu}$ and $L_{\tau}$ were strongly violated during the sphaleron epoch, we would insist that $L_{e}$ is not similarly violated, if we want to keep sphalerons and neutrino mass effects from erasing the baryon asymmetry. Since we are working above the temperature at which the Higgs gets its VEV, however, the effect of interest will be scatterings of leptons into bosons.

By assumption, the $\Delta L=2$ scattering processes $L_{i} L_{j} \rightarrow H H$ will be in equilibrium if $i$ and $j$ are either $\mu$ or $\tau$, which means that in place of the conservation laws for $\frac{1}{3} B-L_{\mu}$ and $\frac{1}{3} B-L_{\tau}$ in eq. (团), we will have two nonstandard equilibrium conditions

$$
\mu_{\mu_{L}}=\mu_{\tau_{L}}=-\mu_{0}
$$

involving the Higgs chemical potential. As in the previous section, the new constraints allow us to solve for the parameters $a$ and $b$ in eq. (15). We find that

$$
\begin{aligned}
\mu_{0} & =\frac{3}{55} \mu_{e_{R}}+\frac{1}{11} \mu_{e_{L}} \\
\mu_{B} & =\frac{24}{165} \mu_{e_{R}}-\frac{12}{11} \mu_{e_{L}} .
\end{aligned}
$$

In addition to the equilibrium conditions (46) for the mu and tau species, the new interaction provides a source of electron-type lepton number violation which goes out of equilibrium at a temperature depending on the size of the neutrino mass matrix elements $m_{e i}$. The effect 
is parametrized by the integrals $I_{\Delta L, i}$ in the Boltzmann equation (10), calculated in section 3. The evolution equations for the two electron chiralities then become

$$
\begin{aligned}
\frac{\mathrm{d} L_{e_{R}}}{\mathrm{~d} t} & =-\Gamma_{R L}\left(\frac{58}{55} L_{e_{R}}-\frac{10}{11} L_{e_{L}}\right) \\
\frac{\mathrm{d} L_{e_{L}}}{\mathrm{~d} t} & =-\Gamma_{\Delta L}\left(\frac{24}{13} L_{e_{L}}+\frac{6}{65} L_{e_{R}}\right)-\frac{157}{390} \frac{\mathrm{d} L_{e_{R}}}{\mathrm{~d} t},
\end{aligned}
$$

where $\Gamma_{R L}$ was defined in (18) and

$$
\begin{aligned}
\Gamma_{\Delta L} & =\Gamma_{\Delta L, e}+\frac{1}{2} \Gamma_{\Delta L, \mu}+\frac{1}{2} \Gamma_{\Delta L, \tau} \\
& =\frac{3 T^{3}}{\pi^{5} v^{4}}\left(5\left|m_{e e}\right|^{2}+3\left|m_{e \mu}\right|^{2}+3\left|m_{e \tau}\right|^{2}\right) .
\end{aligned}
$$

Using the results of section 3, we have integrated these equations starting at high temperatures $T \gg 100 \mathrm{TeV}$ where the $e_{R}$-violating interactions have not yet reached equilibrium. We consider a range of initial conditions for $L_{e_{R}}$, including the largest reasonably imaginable asymmetry in $e_{R}$, which would occur if there were only $e_{R}{ }^{-}$and no $e_{R}{ }^{+}$; then dividing $n_{e_{R}}$ by the total entropy gives $L_{e_{R}}(0) \cong 10^{-2}$. The initial value for $L_{e_{L}}$ is given by solving the chemical potential equations subject to the constraint that the $L_{e}$-violating interactions are in equilibrium: $\mu_{e_{L}}=-\mu_{0}$, as in eq. (46). We find that $L_{e_{L}}=-\frac{1}{20} L_{e_{R}}$ and $B=\frac{1}{5} L_{e_{R}}$ initially (see section 5.1, below). The resulting value of the baryon asymmetry is then determined by the final lepton asymmetries using eq. (47): $B=\frac{24}{165} L_{e_{R}}-\frac{12}{11} L_{e_{L}}$.

In figures $6 \mathrm{a}$ and $6 \mathrm{~b}$ we show the evolution of the asymmetries $L_{e_{R}}, L_{e_{L}}$ and $B$ as a function of the temperature for $m_{t}=m_{H}=100 \mathrm{GeV}$. In figure $6 \mathrm{a}$ we have assumed that the electron lepton number violation has gone out of equilibrium before the $e_{R}-e_{L}$ transitions come into equilibrium. Initially all asymmetries have their high temperature equilibrium values. As the right handed electrons come into equilibrium, the asymmetries relax to their low-temperature equilibrium values, which are of the same order as the asymmetries above the transition region and are calculable in terms of the primordial $L_{e_{R}}$ asymmetry. In figure $6 \mathrm{~b}$ we assume that the neutrino mass parameter

$$
\left\langle m_{e i}\right\rangle^{2} \equiv \frac{5}{3}\left|m_{e e}\right|^{2}+\left|m_{e \mu}\right|^{2}+\left|m_{e \tau}\right|^{2}
$$

\footnotetext{
${ }^{6}$ The relative coefficient of $\left|m_{e e}\right|^{2}$, which was wrong in ref. [7], has been corrected here.
} 
takes the value $\left\langle m_{e i}\right\rangle=5 \mathrm{keV}$. Now the asymmetries fall exponentially within the region where the lepton number violation and the $e_{R}-e_{L}$ transitions (with rate $\Gamma_{e_{R} \leftrightarrow e_{L}}$ ) are both in equilibrium. A new equilibrium is reached above the electroweak phase transition temperature, $T_{c} \simeq 154 \mathrm{GeV}$, as the lepton number violating interactions fall out of equilibrium. The lesson to be learned from figure $6 \mathrm{~b}$ is that, in order to erase the asymmetries, the temperature must fall somewhat below that at which $\Gamma_{e_{R} \leftrightarrow e_{L}} / H=1$, as is often assumed in simple estimates.

To obtain a conservative bound on $\left\langle m_{e i}\right\rangle$, we must assume that the lepton number violation is strong enough to decrease the largest imaginable initial baryon asymmetry down to the smallest value of the present baryon asymmetry $B_{\min } \simeq 4 \times 10^{-11}$ inferred from big bang nucleosynthesis [21]. For the former value we take $B_{\text {initial }}=2 \times 10^{-3}$, corresponding to $L_{e_{R}, p}=10^{-2}$. Moreover, we will assume the least favorable values $m_{t}=90 \mathrm{GeV}$ and $m_{H}=60 \mathrm{GeV}$ for the unknown masses of the top quark and the Higgs boson. In this way one obtains the bound

$$
\left\langle m_{e i}\right\rangle \lesssim 20 \mathrm{keV}
$$

an order of magnitude less stringent than the rough estimate given in our letter [7]. This result can also be inferred from figure 7 , where we show the results of a more complete computation of the bound as a function of the initial asymmetry. We also display the dispersion in the bound that results from the above-mentioned uncertainty in the present baryon asymmetry and the top quark and Higgs boson masses. The shaded area corresponds to the intervals $90 \mathrm{GeV}<m_{t}<300 \mathrm{GeV}, 60 \mathrm{GeV}<m_{H}<1 \mathrm{TeV}$ and $4 \times 10^{-11}<B_{\text {final }}<$ $6 \times 10^{-11}$.

A simple analytic estimate for the bound (51) can be obtained by requiring that the rate of lepton number violation, or more generally, any other baryon or lepton number violation under consideration, becomes comparable to the expansion rate after the time $t_{*}$ defined by

$$
\int_{0}^{t_{*}} \mathrm{~d} t \Gamma_{L R}=\ln \left(B_{\text {initial }} / 4 \times 10^{-11}\right) \simeq 18
$$

using our assumption that $B_{\text {initial }}=2 \times 10^{-3}$. To solve equation (52) for the corresponding temperature $T_{*}$, we use the linear fit for $\Gamma_{L R}$ derived in section 4 . After analytically 
integrating the fit function and expanding in the small parameter $T_{c} / T_{*}$ we find that

$$
T_{*} \simeq 1.3 f\left(x_{H}\right) \mathrm{TeV}
$$

where $f\left(x_{H}\right)$ is as in eq. (43). Again assuming that $m_{t}=90 \mathrm{GeV}$ and $m_{H}=60 \mathrm{GeV}$, we get $x_{H} \simeq 0.41$ and then $T_{*} \simeq 1 \mathrm{TeV}$. Note that while we have so far loosely spoken of $T_{*}$ as the temperature at which $e_{R^{-}} e_{L}$ transitions come into equilibrium, it is actually given by the considerably smaller temperature (53), which is relevant for obtaining the bounds on various baryon or lepton number violating operators. Let us finally note that using (53) in the rough condition for freezeout of the lepton-violating interactions,

$$
\left.\frac{\Gamma_{\Delta L}}{H}\right|_{T=T_{*}}=1
$$

gives a bound $\left\langle m_{e i}\right\rangle \lesssim 21 \mathrm{keV}$, in good agreement with the accurate numerical result (51).

\subsection{Baryon number violating operators}

Apart from neutrino masses, there is a host of possible $(B-L)$-violating interactions that have explicit baryon number violation. To further illustrate the relevance of right-handed electrons for preserving a baryon asymmetry, we will show what happens when several such operators are in equilibrium during some epoch.

It is convenient to rewrite the sphaleron and electric charge constraints on the chemical potentials in the form

$$
\begin{aligned}
\frac{35}{3} H+\frac{1}{2} B & =\frac{1}{2} L_{e}+\frac{2}{3}\left(L_{\mu}+L_{\tau}\right)+\frac{1}{2} L_{e_{R}} \\
\frac{2}{3} H+\frac{3}{4} B & =-\frac{1}{2} L_{e}-\frac{1}{3}\left(L_{\mu}+L_{\tau}\right)+\frac{1}{2} L_{e_{R}}
\end{aligned}
$$

where $H$ is the Higgs boson asymmetry, $L_{e} \equiv 2 L_{e_{L}}+L_{e_{R}}$ is the asymmetry for total electrontype lepton number, and similarly for the other flavors. We have noted that in the minimal standard model, there are three conserved quantities $\frac{1}{3} B-L_{i}$ and one which is approximately conserved at high temperatures, $L_{e_{R}}$. In the absence of new interactions we would solve for the baryon and Higgs chemical potentials in terms of the conserved quantities. 
When sufficiently fast interactions are introduced which violate any of the conservation laws, eqs. (55) must be supplemented by the new chemical equilibrium conditions associated with these interactions. Of course we do not want to break all four of the symmetries, since then the solution to the system of equations would be the vanishing of all asymmetries. We do wish to break the particular linear combination $B-L$, however, since only in this case do we get anything new from our observations concerning right-handed electrons. If $B-L$ is conserved during the epoch when sphalerons are in equilibrium, yet has a nonzero value, this by itself is enough to insure a nonvanishing baryon asymmetry, regardless of the electron «4.

For example, consider the $\Delta B=2$ operator

$$
M^{-5}\left(\bar{u}_{R} d_{R}^{c}\right)\left(\bar{d}_{R} d_{R}^{c}\right)\left(\bar{u}_{R} d_{R}^{c}\right)
$$

It conserves the linear combination

$$
2 L_{e}-L_{\mu}-L_{\tau} \equiv C
$$

(Although it conserves both $L_{e}-L_{\mu}$ and $L_{e}-L_{\tau}$ separately, only the sum of these is relevant since $L_{\mu}$ and $L_{\tau}$ enter eqs. (55) in the combination $L_{\mu}+L_{\tau}$.) It also leads to the equilibrium constraint

$$
2 \mu_{u_{R}}+4 \mu_{d_{R}}=0 \Rightarrow B=4 H,
$$

where we used eq. (11) to eliminate the chemical potentials of the right-handed quarks in favor of $\mu_{q_{L}}$ and the relation $\mu_{B}=12 \mu_{q_{L}}$. It is now possible to solve for $B$ and the total lepton number $L$ using eqs. (55,57-58), and in particular their difference

$$
B-L=\frac{3}{68}\left(C-9 L_{e_{R}, p}\right),
$$

where we subscripted $L_{e_{R}}$ to emphasize that it retains its primordial value until $T_{*}$, the temperature at which the rate of $e_{R}$-violation starts to exceed the expansion rate of the universe. Let us suppose that the new interaction (56) goes out of equilibrium at some temperature $T^{\prime}$ below the baryogenesis scale but above $T_{*}$. Then for $T^{\prime}>T>T_{*}$, the

\footnotetext{
${ }^{7}$ This is similar to the result in ref. [7] but written in terms of the conserved quantities $C$ and $L_{e_{R}}$.
} 
asymmetry $B-L$ maintains its equilibrium value (59), since nothing occurs that would change it. As the temperature falls below $T_{*}, B-L$ continues to be conserved, even though $L_{e_{R}}$ itself changes. At this point, the complete equilibrium conditions assumed by Harvey and Turner prevail, and we can use their results to determine the final $B$ in terms of $B-L$.

This result is remarkable in two respects. First, $B-L$ in (59) is completely independent of its initial value, at temperatures before the $\Delta B=2$ operator came into equilibrium. One possibility is that it was initially zero, in which case the new operator actually worked in conjunction with sphalerons to create the baryon asymmetry, which would have been zero in its absence. So we have here a new way to convert a primordial lepton asymmetry into baryons. Secondly, even though this mechanism depends on the conservation of $e_{R}$ number, it does not require that $L_{e_{R}}$ be nonzero. It was enough to have an asymmetry between the different flavors of leptons in this example.

In general we can violate all three of the quantities $\frac{1}{3} B-L_{i}$ and still obtain a nontrivial solution for $B$. For our second example, we take the operators

$$
M^{-5}\left(\bar{u}_{R} u_{R}{ }^{c}\right)\left(\bar{u}_{R} \ell_{i, R}^{c}\right)\left(\bar{L}_{j} \tau_{2} L_{k}^{c}\right),
$$

where the indices $i, j, k$ are generational and $\tau_{2}$ is the Pauli matrix. Although there are numerous possible combinations of $i, j, k$, consider at first any three which break all three symmetries. These give relations among the chemical potentials which as before can be expressed entirely in terms of left-handed fields using the constraints (11) from Higgs boson interactions to eliminate the right-handed fields. After so doing, the new relations are

$$
3 \mu_{q_{L}}=-\mu_{\mu_{L}}=-\mu_{\tau_{L}}=\mu_{0}
$$

which translate directly to

$$
B=-L_{\mu}=-L_{\tau}=4 H
$$

in terms of the asymmetries. We can solve these together with eqs. (55) for all of the asymmetries in terms of $L_{e_{R}}$ :

$$
H=\frac{1}{20} L_{e_{R}} ;
$$




$$
\begin{aligned}
B & =-L_{\mu}=-L_{\tau}=\frac{1}{5} L_{e_{R}} \\
L_{e} & =\frac{9}{10} L_{e_{R}} .
\end{aligned}
$$

The final baryon asymmetry will again be determined by $B-L$, whose equilibrium value above $T_{*}$ is

$$
B-L=-\frac{3}{10} L_{e_{R}, p}
$$

as first shown in [7].

If the primordial asymmetry in $e_{R}$ was sufficiently large, the new $B$ - and $L$-violating interactions (56,60) were harmless for the baryon asymmetry, so long as they fell out of equilibrium above $T_{*}$. As outlined in the introduction to this section, this leads to upper bounds on the masses in their coefficients [5]. Let us examine the bound for the dimension nine operators $M^{-5} q q q q q q$ and $M^{-5} q q q l l l$ we have just discussed. For a particular scattering channel, say for a $3 \rightarrow 3$ process, we saw in section 2 that the relevant rate is typically given by $\Gamma \sim 12 I / T^{3}$, where

$$
I \cong \int \prod_{i=i}^{6} \mathrm{~d} \prod_{i}(2 \pi)^{4} \delta\left(p_{1}+p_{2}+p_{3}-p_{4}-p_{5}-p_{6}\right)|\mathcal{M}|^{2} e^{-\beta\left(E_{1}+E_{2}+E_{3}\right)}
$$

and we can estimate the matrix element as

$$
\begin{aligned}
|\mathcal{M}|^{2} & =8 M^{-10} p_{1} \cdot p_{2} p_{3} \cdot p_{4} p_{5} \cdot p_{6} \\
& \cong 8 M^{-10} \prod_{i} E_{i} .
\end{aligned}
$$

There are $6 \cdot 5 \cdot 4$ different channels for the $3 \rightarrow 3$ processes and $6 \cdot 4$ for the $2 \rightarrow 4$ processes. Putting everything together and evaluating the integrals, we find a rate of

$$
\Gamma \cong 288(2 / \pi)^{14} T\left(\frac{T}{M}\right)^{10}
$$

which, if we insist it be less than the Hubble rate at temperature $T_{0}$, gives the lower limit on $M$ of

$$
M>0.7 T_{0}\left(\frac{M_{\mathrm{Pl}}}{T_{0}}\right)^{1 / 10}
$$


in remarkably good agreement with the dimensional analysis estimate (45). Previous limits on this operator [5] ranged from $10^{3}-10^{12} \mathrm{GeV}$ for $T_{0}$ between $10^{2}-10^{12} \mathrm{GeV}$. We have shown that the appropriate temperature is $T_{0}=T_{*} \sim 1 \mathrm{TeV}$. The difference for the bound on the scale of new physics is marked:

$$
M>\left\{\begin{array}{ll}
4 \times 10^{12} \mathrm{GeV}, & T_{0}=10^{12} \mathrm{GeV} \\
30 \mathrm{TeV}, & T_{0}=T_{*}
\end{array} .\right.
$$

and hence the limit is much closer to the weaker of the bounds given in [5]. Limits on other nonrenormalizable operators are similarly weakened.

The reader may wonder how general our results are. Did we need exactly three of the operators (60) in equilibrium, and did it matter which three? Intuitively we expect that the answer to both questions is no as long as all three symmetries $\frac{1}{3} B-L_{i}$ are broken, since the only thing keeping the chemical potentials from vanishing is the necessity of maintaining charge neutrality of the universe by canceling the charge of the conserved $e_{R}$ asymmetry. Therefore it should not matter how many or which kind of operators do the job of breaking $\frac{1}{3} B-L_{i}$.

It is easy to prove that having other operators in addition to all those we have already considered has no effect on the equilibrium conditions (63). Imagine some operator which is perfectly arbitrary except that it conserves $e_{R}$ number. It can be characterized by a set of integers $n_{F}$, the net number of fields of type $F$, defined to be the number of fields $F$ minus the number of charge-conjugated fields $F^{c}$. If it is in equilibrium, it gives a condition on the chemical potentials,

$$
\sum_{F} n_{F} \mu_{F}=0
$$

But the coefficients $n_{F}$ must be such that the total hypercharge of the operator vanishes, because of gauge invariance:

$$
\sum_{F} Y_{F} n_{F}=0 .
$$

Now note that if the solution for the chemical potentials takes the form $\mu_{F}=c Y_{F}$ 22], then these equations are consistent with each other. In this case, eq. (70) is automatically satisfied, so it is not really a new condition, as we set out to demonstrate. It is easy to 
verify from eqs. (63) that the chemical potential of each particle is indeed proportional to its hypercharge. Therefore we can add as many new operators as we want: as long as they conserve $e_{R}$ number, they lead to the same equilibrium conditions, which can be written as

$$
\mu_{A}=\frac{1}{20} Y_{A} \mu_{e_{R}}
$$

\section{Summary and Conclusions}

Although the smallness of the electron Yukawa coupling is not completely understood, its smallness is an experimental fact. In the absence of new physics beyond the standard model and still below the GUT scale for which the right-handed electron is not a singlet, $e_{R}$ remains an essentially decoupled state for a good part of the early evolution of the Universe. Indeed we have accurately determined the decoupling temperature of this state to be $T_{*}=1.3 f\left(x_{H}\right)$ $\mathrm{TeV}$.

Our interest in the $e_{R}$ decoupling temperature is directly related to the preservation and generation of the cosmological baryon asymmetry. We have shown conclusively (up to mass effects [20, 19]) that in a $B-L=0$ universe (with $B-L$ conserved) sphaleron erasure of a primordial asymmetry does indeed occur in the standard model. The final asymmetry in this case is exponentially sensitive to model parameters and it may be possible to preserve the asymmetry in this case in an extension of the standard model.

Even in models which do not conserve $B-L$, sphaleron erasure of a baryon asymmetry may occur if other $B / L$-violating interactions are included in any extension of the standard model. A simple example of such an interaction is the $\Delta L=2$ operator induced from neutrino mass generation via the seesaw mechanism. The preservation of the baryon asymmetry places restrictions on such operators and thus on the neutrino mass matrix. In the literature several widely differing bounds on neutrino masses have been given. Here we have clarified these bounds and shown again the importance of the $e_{R}$ decoupling. In particular we have shown that erasure may occur only between $T_{*}$ and the electroweak phase transition temperature, $T_{c}$. We have also shown how the role of $e_{R}$ decoupling should be applied to bounds on other $B / L$ violating operators. 
Finally, we have demonstrated that because of $e_{R}$ decoupling, an initial lepton or $e_{R}$ asymmetry can be used to generate a baryon asymmetry even when $B=0$ initially. In such cases, not only do we drastically weaken upper bounds on new operators, but even imply a lower bound such that they would be able to induce a nonzero $B-L$ and ultimately the baryon asymmetry.

\section{Acknowledgements}

We would like to thank Sonia Paban for useful discussions. This work was supported in part by DOE grant DE-AC02-83ER-40105. The work of KAO was in addition supported by a Presidential Young Investigator Award and the work of KK was in addition supported by The Finnish Academy. 


\section{References}

[1] V. Kuzmin, V. Rubakov and M. Shaposhnikov, Phys. Lett. B155 (1985) 36.

[2] P. Arnold and L. McLerran, Phys. Rev. D36 (1987) 581, Phys. Rev. D37 (1988) 1020.

[3] M. Fukugita and T. Yanagida, Phys. Rev. D42 (1990) 1285.

[4] Harvey and M. Turner, Phys. Rev. D42 (1990) 3344.

[5] B.A. Campbell, S. Davidson, J. Ellis, and K.A. Olive, Phys. Lett. B256 (1991) 457; Astroparticle Physics 1 (1992) 77.

[6] W. Fischler, G.F. Giudice, R.G. Leigh, and S. Paban, Phys. Lett. B258 (1991) 45.

[7] J.M. Cline, K. Kainulainen and K.A. Olive, Phys. Rev. Lett. 71 (1993) 2372.

[8] B.A. Campbell, S. Davidson, J. Ellis, and K.A. Olive, Phys. Lett. B297 (1992) 118 (CDEO).

[9] M.E. Shaposhnikov, in Electroweak Interactions and Unified Theories; proceedings of the XXVIIth Rencontres de Moriond (1992, Editions Frontieres, France).

[10] J.M. Cline, K. Kainulainen and K.A. Olive, Astroparticle Physics 4 (1993) 387.

[11] H.A. Weldon, Phys. Rev. D26 (1982) 2789.

[12] M.E. Carrington, Phys. Rev. D45 (1992) 2933;

J.I. Kapusta, Finite-temperature field theory, (1989, Cambridge University Press) 177.

[13] E.W. Kolb and S. Wolfram, Nucl. Phys. B172 (1980) 224.

[14] J.N. Fry, K.A. Olive, and M. Turner, Phys. Rev. D22 (1980) 2953, 2977.

[15] L.E. Ibáñez and F. Quevedo, Phys. Lett. B283 (1992) 261.

[16] J. Ellis, K. Enqvist, D.V. Nanopoulos, and K.A. Olive, Phys. Lett. B191(1987) 343. 
[17] B. Campbell, S. Davidson, and K.A. Olive, Nucl. Phys. B399 (1993) 111.

[18] A. Nelson and S. Barr, Phys. Lett. B246 (1990) 141.

[19] H. Dreiner and G.G. Ross, Oxford preprint OUTP-92-08P (1992).

[20] V. Kuzmin, V. Rubakov and M. Shaposhnikov, Phys. Lett. B191 (1987) 171.

[21] T.P Walker, G.Steigman, D.N. Schramm, K.A. Olive, and H.-S. Kang, Ap.J. 376 (1991) 51.

[22] A. Antaramian, L.J. Hall and A. Rašin, Lawrence Berkeley Laboratory preprint LBL34827 (1993). 


\section{Figure Captions}

Fig. 1 (a) Higgs boson decay into right-handed electron and lepton doublet; Higgs and gauge boson scattering of (b) $L_{e}$ into $e_{R}$ and (c) bosons into $L_{e}$ and $e_{R}$; lepton number violating scatterings of $(\mathrm{d}) e_{L} \ell^{-}$or $e_{L} \nu_{\ell}$ into Higgs bosons and (e) $e_{L}$-Higgs into $(\ell$ or $\left.\nu_{\ell}\right)$-Higgs.

Fig. $2 m_{H}(T) / T$ as a function of the zero temperature Higgs boson mass $m_{0}$ for top quark masses of $180 \mathrm{GeV}$ (top curve), $135 \mathrm{GeV}$ (middle curve) and $90 \mathrm{GeV}$ (lower curve).

Fig. 3 (a) s-channel scattering of third-generation quark doublet and right-handed top antiquark into $e_{R}$ and $L_{e}^{c}$; (b) t-channel scattering processes.

Fig. 4 Diagrams contributing to $e_{R}$ production by Higgs and gauge boson scattering. The first two are present for both $B$ and $W$ gauge bosons, whereas the last exists only for $B$.

Fig. 5 a) Contours of constant $B_{\text {final }} / B_{e q}$, when the scattering contribution to the chirality changing rate is omitted. Solid lines correspond to the present work; for comparison the previous result of CDEO is shown by the dashed lines. b) The solid lines are contours of constant $B_{\text {final }} / B_{e q}$ with the full interaction rate, including scatterings. Dashed lines are contours of constant thermal Higgs mass parameter $x_{H}$.

Fig. 6 a) Evolution of the asymmetries $L_{e_{R}}, L_{e_{L}}$ and $B$ as a function of temperature, when electron lepton number is conserved at low temperatures, using the initial value $L_{e_{R}, p}=$ $10^{-3}$. b) Evolution of absolute values of asymmetries with electron lepton number violation corresponding to $\left\langle m_{e i}\right\rangle=5 \mathrm{keV} . B$ is initially negative and the dip in the corresponding curve marks the place where $B$ becomes positive. Similarly $L_{e_{L}}$ changes sign from positive to negative. $L_{e_{R}}$ remains negative over the whole range. 
Fig. 7 The shaded area between the curves $\mathrm{C}$ and $\mathrm{D}$ shows the region consistent with the observed baryon asymmetry for some choice of parameters $m_{t}$ and $m_{H}$. Curve $\mathrm{C}$ corresponds to $B, m_{t}, m_{H}=4 \times 10^{-11}, 90 \mathrm{GeV}, 60 \mathrm{GeV}$ and curve D to $B, m_{t}, m_{H}=$ $6 \times 10^{-11}, 90 \mathrm{GeV}, 1 \mathrm{TeV}$. 

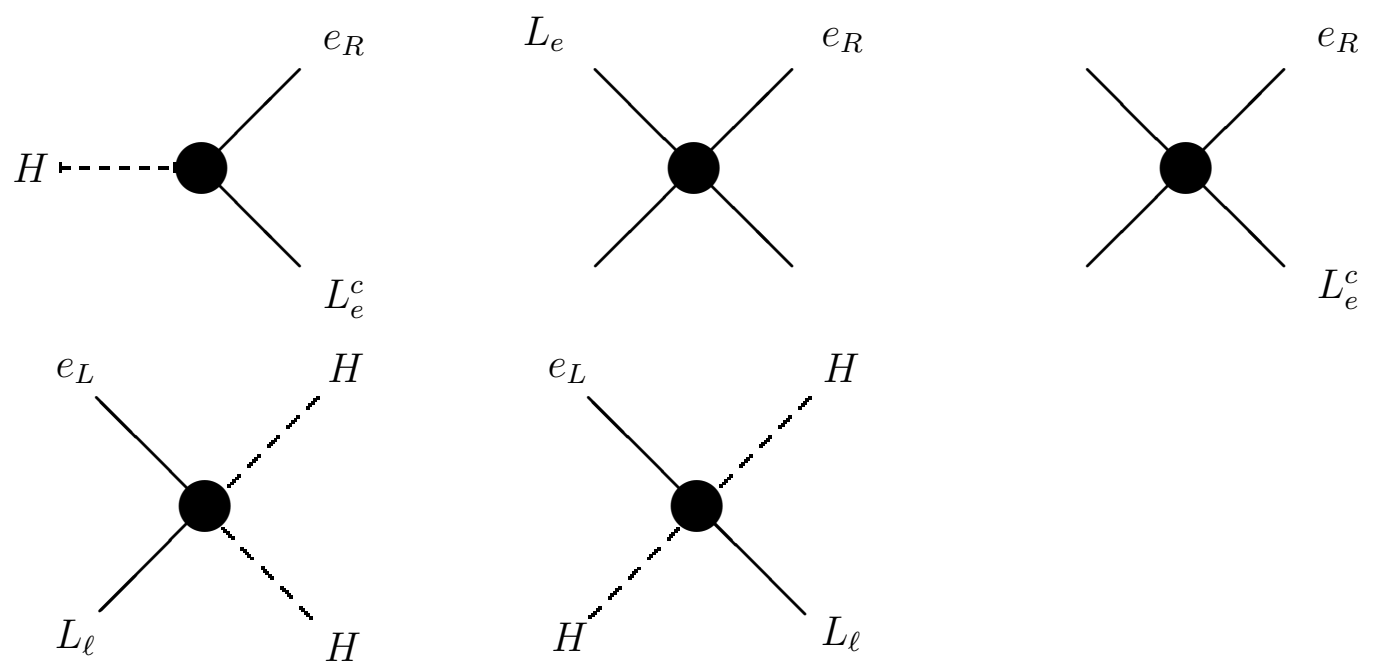

Figure 1

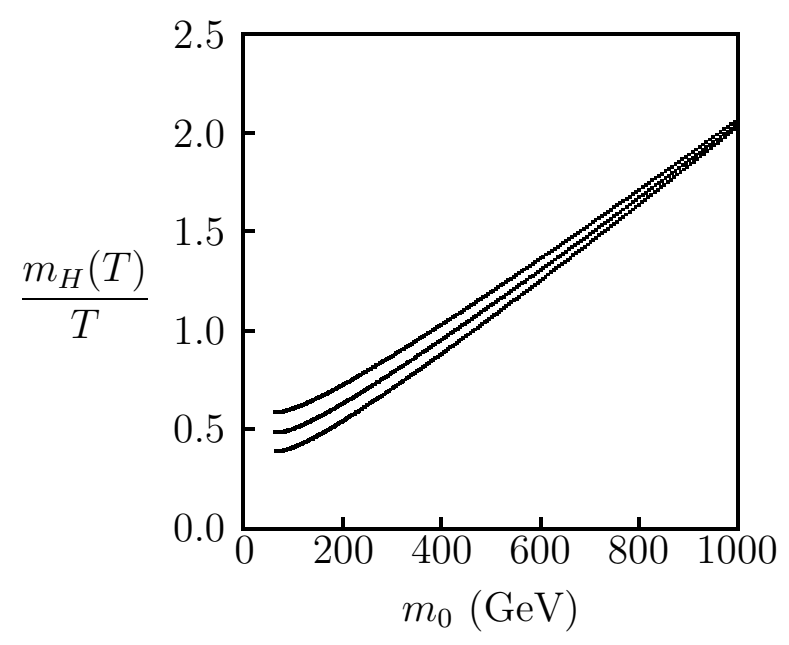

Figure 2
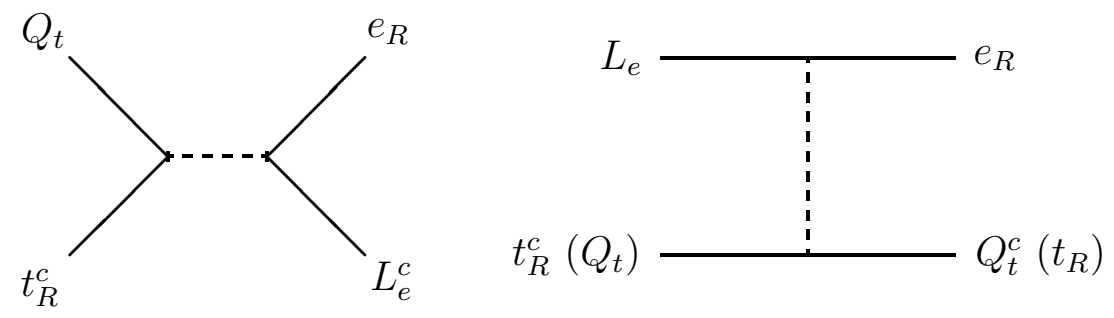

Figure 3 

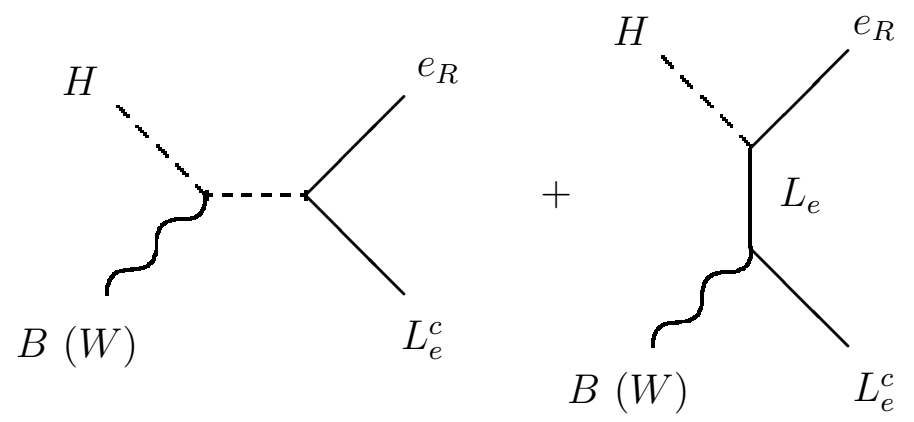

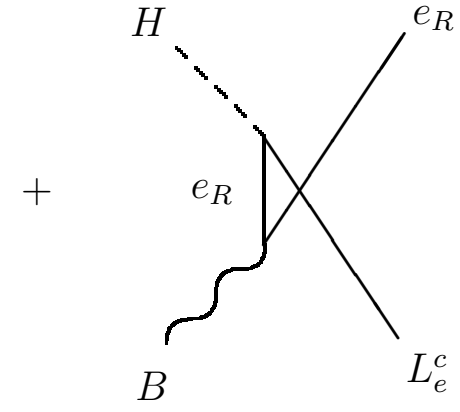

Figure 4 
This figure "fig1-1.png" is available in "png" format from: http://arxiv.org/ps/hep-ph/9401208v1 
This figure "fig1-2.png" is available in "png" format from: http://arxiv.org/ps/hep-ph/9401208v1 
This figure "fig1-3.png" is available in "png" format from: http://arxiv.org/ps/hep-ph/9401208v1 